\section{Parametric Macromodeling of Linear High-Frequency Systems using Multiple Frequency Scaling and Sequential Sampling}

\section{K. Chemmangat, F. Ferranti, T. Dhaene and L. Knockaert}

\begin{abstract}
This letter presents an enhanced parametric macromodeling scheme for linear high-frequency systems based on the use of multiple frequency scaling coefficients and a sequential sampling algorithm to fully automate the entire modeling process. The proposed method is applied on a ring resonator bandpass filter example and compared with another state-ofthe-art macromodeling method to show its improved modeling capability and reduced setup time.
\end{abstract}

Introduction: Design activities of electromagnetic (EM) systems such as design space exploration, optimization, sensitivity analysis, etc., often require a substantial number of computationally expensive EM simulations. The development of parametric macromodels acting as accurate and efficient surrogate models for complex EM systems is an active field of research [1, 2, 3, 4]. These models tend to be good approximations of the EM system behavior, characterized by frequency and additional design parameters (such as geometrical or substrate features) and can be used to speed-up the design process. Robust interpolation-based parametric macromodeling methods have been proposed over the recent years, based on the parameterization of a set of frequency-dependent rational models called root macromodels [1], [4]. In [4], interpolation of root macromodels at the input-output level, based on two scaling coefficients was presented : one of the coefficients is a multiplicative factor at the input/output level of the system (amplitude scaling) and the other coefficient is a compression or expansion term for the Laplace variable $s$ (frequency scaling). The approach of [4] results in high modeling capability and robustness.

In this letter, the parametric macromodeling method proposed in [4] is generalized by using multiple frequency scaling coefficients for all partial fractions of the root macromodels. This allows to model the behavior of the partial fractions of the root macromodels independently, in order to achieve a more flexible modeling capability. The proposed method is compared with the approach described in [4] to show its enhanced modeling capability and reduced CPU setup time.

Proposed Parametric Macromodeling Method: The proposed parametric macromodeling scheme starts from a set of multivariate data samples $\left\{\left(s_{n}, \vec{g}_{k}\right), \mathbf{H}\left(s_{n}, \vec{g}_{k}\right)\right\}, n=1, \ldots, N_{s}, k=1, \ldots, K^{\text {tot }}$, which depend on the complex frequency $s=j \omega$ and additionally $N$ design variables in a vector form $\vec{g}=\left(g^{(1)}, \ldots, g^{(N)}\right)$. The design space $\Omega(\vec{g})$ consists of $N$ dimensional hyper-rectangular $(N$-box $)$ regions $\Omega_{l}, l=1, \ldots, L$ such that $\Omega(\vec{g})=\cup \Omega_{l}$. Each of these $\Omega_{l}$ is defined by $2^{N}$ corner root macromodels, identified from the data samples $\mathbf{H}\left(s, \vec{g}_{i}{ }^{\Omega_{l}}\right)$ using the well known Vector Fitting (VF) technique [5] in pole-residue form:

$$
\mathbf{R}^{\Omega_{l}}\left(s, \vec{g}_{i}^{\Omega_{l}}\right)=\sum_{p=1}^{P_{i}^{\Omega_{l}}} \frac{C_{p, i}^{\Omega_{l}}}{s-a_{p, i}^{\Omega_{l}}}+D_{i}^{\Omega_{l}} ; i=1, \ldots, 2^{N}
$$

Here matrices $C_{p, i}^{\Omega_{l}}$ represent the residues, $a_{p, i}^{\Omega_{l}}$ are the poles and $D_{i}^{\Omega_{l}}$ are constant matrices. In the next step each of these $N$-box regions $\Omega_{l}$ is parameterized. For clarity, let us consider a two variable $(N=2)$ design space region $\Omega_{l}$ defined by four corners $\vec{g}_{1}{ }^{\Omega_{l}}=\left(g_{1}^{1}, g_{2}^{1}\right), \vec{g}_{2}{ }^{\Omega_{l}}=\left(g_{1}^{2}, g_{2}^{1}\right)$, $\vec{g}_{3}{ }^{\Omega_{l}}=\left(g_{1}^{1}, g_{2}^{2}\right)$, and $\vec{g}_{4}{ }^{\Omega_{l}}=\left(g_{1}^{2}, g_{2}^{2}\right)$ as in Fig. 1 with associated corner root macromodels $\mathbf{R}^{\Omega_{l}}\left(s, \vec{g}_{i}\right), i=1, \ldots, 4$. It should be noted however that this approach is not restricted to $N=2$, but can be applied for any dimension $N$. Also, for simplicity, we omit the superscript $\Omega_{l}$ for further discussions in this section. In [4], the amplitude scaling and frequency

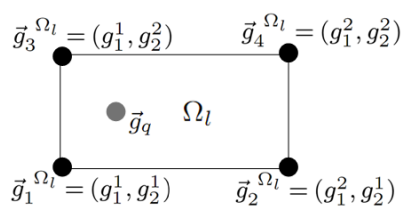

Fig. 1. A two dimensional design space with four root macromodels. scaling coefficients $\left(\alpha_{1}, \alpha_{2}\right)$ are calculated using the optimization

$$
\left(\alpha_{1, i j}^{*}, \alpha_{2, i j}^{*}\right)=\underset{\left(\alpha_{1, i j}, \alpha_{2, i j}\right)}{\arg \min }\left[\operatorname{Err}\left(\tilde{\mathbf{R}}_{j}\left(s, \vec{g}_{i}\right), \mathbf{H}\left(s, \vec{g}_{j}\right)\right)\right] .
$$

In (2), $\tilde{\mathbf{R}}_{j}\left(s, \vec{g}_{i}\right)=\alpha_{1, i j} \mathbf{R}\left(s \alpha_{2, i j}, \vec{g}_{i}\right)$, is the modified response of $\mathbf{R}\left(s, \vec{g}_{i}\right)$ to match $\mathbf{H}\left(s, \vec{g}_{j}\right)$ and $\operatorname{Err}(\cdot)$ is a suitable error measure [4].

In this letter, the frequency scaling coefficients $\alpha_{2, i j}$ are further refined and improved by defining a separate frequency scaling coefficient $\beta_{p, i j}$ for every term $p$ in the rational model in a pole-residue form (1). The modified $\tilde{\mathbf{R}}_{j}\left(s, \vec{g}_{i}\right)$ is given by:

$$
\tilde{\mathbf{R}}_{j}\left(s, \vec{g}_{i}\right)=\alpha_{1, i j} \sum_{p=1}^{P_{i}} \frac{C_{p, i}}{s \beta_{p, i j}-a_{p, i}}+D_{i}
$$

The optimal $\beta_{p, i j}, p=1, \ldots, P_{i}$ are found by performing an optimization step similar to (2) with $\alpha_{2, i j}^{*}$ from (2) as initial value for all $\beta_{p, i j}$. For complex-conjugate pole pairs $\left(p_{1}, p_{2}\right)$ the same coefficient $\beta_{p_{1}, i j}=\beta_{p_{2}, i j}$ is used for both poles to preserve symmetry, which reduces the overall number of coefficients to be optimized. The evaluation of the model at a generic point $\vec{g}_{q}$ in the design space (Fig. 1) is done similarly to [4] as:

i For each corner root macromodel of the cell containing $\vec{g}_{q}, \mathbf{R}\left(s, \vec{g}_{i}\right), i=$ $1, \ldots, 2^{N}$, the amplitude scaling coefficient $\alpha_{1, i j}$ and frequency scaling coefficients $\beta_{p, i j}, p=1, \ldots, P_{i}$ are interpolated using a multilinear interpolation [6] over $\vec{g}$ at the point $\vec{g}_{q}$ to find $\alpha_{1, i q}$ and $\beta_{p, i q}, p=$ $1,2, \ldots P_{i}$. This results in the modified root macromodels, $\tilde{\mathbf{R}}_{q}\left(s, \vec{g}_{i}\right)=$ $\alpha_{1, i q} \sum_{p=1}^{P_{i}} \frac{C_{p, i}}{s \beta_{p, i q}-a_{p, i}}+D_{i}$ at $\vec{g}_{q}$,

ii Then, $\tilde{\mathbf{R}}_{q}\left(s, \vec{g}_{i}\right)$, are further interpolated using the multilinear interpolation [6] over $\vec{g}$ to get the final model at the points $\vec{g}_{q}, \mathbf{R}\left(s, \vec{g}_{q}\right)$.

This parametric macromodeling approach is performed for each region $\Omega_{l}$ to cover the complete design space.

Sequential Sampling Algorithm: A sequential sampling scheme can be used to automatically build parametric macromodels using the parametric macromodeling method discussed above. In this letter, the grid-based sequential sampling algorithm [7] is used to adaptively sample the design space. The sampling scheme consists of the following steps:

I Define the initial $N$-box region of the design space $\Omega_{l}$, (initially $l=$ $L=1$ ) with $N$ design variables $\vec{g}=\left(g^{(1)}, \ldots, g^{(N)}\right)$,

II construct the parametric macromodel $\mathbf{R}^{\Omega_{l}}(s, \vec{g})$ for $\Omega_{l}$ with the $2^{N}$ corner points using the approach described above,

III for each $N$-box region $\Omega_{l}, l=1, \ldots, L$, check the error threshold $\Delta$ at the center of the maximum dynamic edge (find as in [7]) by comparing the macromodel evaluation with the actual EM simulation,

i. IF: $\left(\operatorname{Err}_{\Omega_{l} \text {,edge }}>\Delta\right)$, Divide $\Omega_{l}$ into two subregions along the maximum dynamic edge, update $L=L+1, l=l+1$ and go to Step II.

ii. ELSE IF: $\left(E r r_{\Omega_{l}}\right.$,edge $\left.<=\Delta\right)$, check also the error at the geometric center of $\Omega_{l}$ and if $\operatorname{Err}_{\Omega_{l} \text {,center }}>\Delta$, divide into two subregions along the maximum dynamic edge, update $L=L+1, l=l+1$ and go to Step II.

iii. ELSE IF: $\left(E r r_{\Omega_{l}, \text { edge }}<=\Delta\right.$ and $\left.E r r_{\Omega_{l}, \text { center }}<=\Delta\right)$, increment $l=l+1$. IF $(l \leq L)$ : Not all regions $\Omega_{l}$ are checked for the error criteria, go to Step III; ELSE: Termination

Numerical Example: The scattering parameter response of a ring resonator bandpass filter (see Fig. 2) has been modeled. ADS Momentum ${ }^{1}$ has been used as EM solver. The substrate has a relative permittivity $\epsilon_{r}=4.32$, a loss tangent $\delta=0.002$ and a thickness equal to $1.52 \mathrm{~mm}$. The lengths $L_{1} \in$ $[20.0,23.0] \mathrm{mm}, L_{2} \in[20.0,23.0] \mathrm{mm}$ and the spacing $S_{1} \in[0.05,0.3]$ $\mathrm{mm}$ (see Fig. 2) are chosen as three design variables in addition to frequency $\in[1.0,3.0] \mathrm{GHz}$. The parametric behavior of the filter is shown in Fig. 3. For the sequential sampling algorithm the Mean Absolute Error (MAE) measure per port is used to assess the accuracy of the parametric macromodel in every $N$-box region of the design space:

$$
E^{\mathrm{MAE}}(\vec{g})=\max _{\substack{u=1, \ldots, P_{\text {in }} \\ v=1, \ldots, P_{\text {out }}}} \frac{1}{N_{s}}\left(\sum_{n=1}^{N_{s}}\left|R_{u, v}\left(s_{n}, \vec{g}\right)-H_{u, v}\left(s_{n}, \vec{g}\right)\right|\right)
$$

where the EM simulation response $H_{u, v}(s, \vec{g})$ is compared with the parametric macromodel response $R_{u, v}(s, \vec{g})$, and $P_{i n}$ and $P_{\text {out }}$ are the

${ }^{1}$ Momentum EEsof EDA, Agilent Technologies, Santa Rosa, CA. 


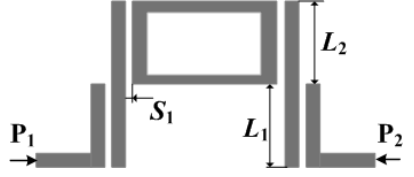

Fig. 2. Layout of the ring resonator bandpass filter.

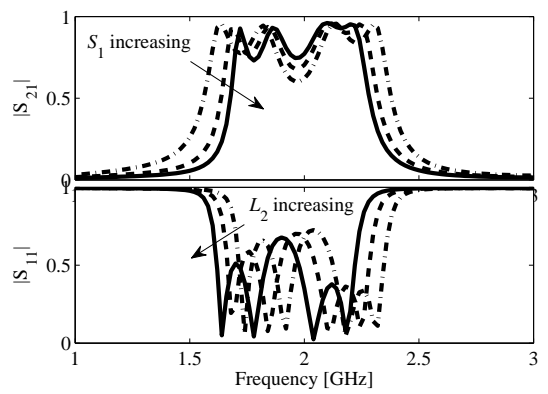

Fig. 3. Magnitude of $\mathbf{S}_{21}$ and $\mathbf{S}_{11}$ as a function of $S_{1}$ and $L_{2}$.

number of input and output ports, respectively. The new parametric macromodeling method as well as the recent method in [4] have been used along with the grid-based sequential sampling algorithm [7] to build accurate parametric macromodels. The MAE measure (4) was kept at a target accuracy of $\Delta=-40 \mathrm{~dB}$ for the sequential sampling. Some comparison results are shown in Table 1 . The total number of generation (Gen.) and validation (Val.) points are shown in the table. As it can be seen, the new method needs significantly less data samples for the same accuracy target, proving its improved modeling capability. The overall CPU time to build the corresponding model is consequently reduced.

Table 1: Comparison: proposed method versus the method of [4]

\begin{tabular}{|c|c|c|c|c|c|}
\hline \multirow{2}{*}{ Method } & \multicolumn{2}{|c|}{ \# Samples } & \multicolumn{2}{c|}{ CPU Time } & \multirow{2}{*}{$\begin{array}{c}\text { Accuracy } \\
\text { [dB] }\end{array}$} \\
\cline { 2 - 5 } & Gen. & Val. & Modeling & Data Gen. & -40.4 \\
\hline proposed & 32 & 18 & $6 \mathrm{~m} 56 \mathrm{~s}$ & $36 \mathrm{~m} 41 \mathrm{~s}$ & -40.1 \\
\hline$[4]$ & 116 & 86 & $22 \mathrm{~m} 30 \mathrm{~s}$ & $2 \mathrm{~h} 31 \mathrm{~m} 29 \mathrm{~s}$ & -40.1 \\
\hline
\end{tabular}

Fig. 4 shows the distribution of design space points selected using the two parametric macromodeling methods. The method [4] finds more difficult to model the filter behavior with respect to the design parameters and needs significantly larger number of points along every design space dimension. Fig. 5 compares the magnitude of $\mathbf{S}_{21}$ between the EM solver and the proposed parametric macromodel for three random validation points. The different responses show a very good agreement.

Conclusion: We have presented an enhanced parametric macromodeling method for linear high-frequency systems. It is combined with a sequential sampling scheme is able to generate accurate parametric macromodels in an efficient and fully automated way. A comparison is made with state-ofthe-art modeling approach on a pertinent numerical example to show the improved modeling capability and efficiency of the new method.

Acknowledgment: This work was supported by the Research Foundation Flanders (FWO) and by the Interuniversity Attraction Poles Programme BESTCOM initiated by the Belgian Science Policy Office. Francesco Ferranti is currently an FWO Post-Doctoral Research Fellow with the Department of Information Technology (INTEC), Ghent University, Ghent, Belgium.

K. Chemmangat, F. Ferranti, T. Dhaene and L. Knockaert (Ghent University-iMinds, Gaston Crommenlaan 8/201, B-9050 Gent, Belgium)

E-mail: krishnan.cmc@intec.ugent.be

\section{References}

1 F. Ferranti, L. Knockaert, and T. Dhaene, "Parameterized S-parameter based macromodeling with guaranteed passivity," IEEE Microwave and Wireless Component Letters, vol. 19, no. 10, pp. 608-610, Oct. 2009.

2 P. Triverio, M. Nakhla, and S. Grivet-Talocia, "Passive parametric macromodeling from sampled frequency data," IEEE workshop on Signal Propagation and Interconnects, pp. 117 -120, May 2010.

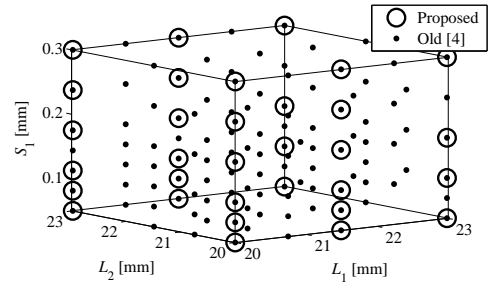

Fig. 4. Model generation points in the design space

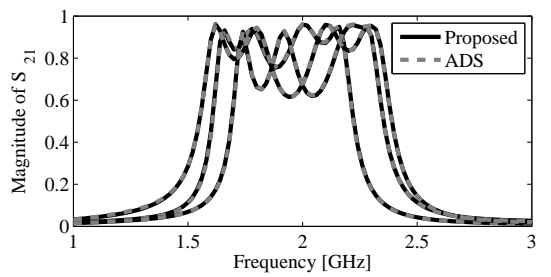

Fig. 5. Magnitude of $\mathbf{S}_{21}$ at three random validation points.

3 F. Ferranti, L. Knockaert, T. Dhaene, and G. Antonini, "Passivitypreserving parametric macromodeling for highly dynamic tabulated data based on Lur'e equations," IEEE Trans. on Microwave Theory and Techniques, vol. 58, no. 12, pp. 3688 -3696, Dec. 2010.

4 - "Parametric macromodeling based on amplitude and frequency scaled systems with guaranteed passivity," Int. J. of Numerical Modelling: Electronic Networks, Devices and Fields, vol. 25, no. 2, pp. 139-151, 2012.

5 B. Gustavsen and A. Semlyen, "Rational approximation of frequency domain responses by vector fitting," IEEE Trans. on Power Delivery, vol. 14, no. 3, pp. 1052-1061, July 1999.

6 A. Weiser and S. E. Zarantonello, "A note on piecewise linear and multilinear table interpolation in many dimensions," Mathematics of Computation, vol. 50, no. 181, pp. 189-196, Jan. 1988.

7 K. Chemmangat, F. Ferranti, T. Dhaene, and L. Knockaert, "Scalable models of microwave system responses using sequential sampling on unstructured grids," Int. J. of Numerical Modelling: Electronic Networks, Devices and Fields, 2013. doi: http://dx.doi.org/10.1002/jnm.1903 